\title{
Loss of Expression of the PTEN Gene Protein Product Is Associated with Poor Outcome in Breast Cancer
}

\author{
Peter L. Depowski, M.D., Seth I. Rosenthal, M.D., Jeffrey S. Ross, M.D. \\ Department of Pathology and Laboratory Medicine, Albany Medical College, Albany, New York
}

\begin{abstract}
Introduction: The PTEN gene, a candidate tumor suppressor, is localized to chromosome 10q23 and shares extensive homology with cytoskeletal proteins auxilin and tensin. A high frequency of mutations at the PTEN locus has been described in a variety of neoplasms including breast cancer. However, the role of PTEN alternations and its association with outcome variables in breast neoplasia is not well established. Design: Formalin-fixed paraffin embedded tissues from 151 women (mean age 62 years, range 26-98) with primary diagnosis of invasive breast cancer were evaluated for PTEN protein expression by automated immunohistochemical methods. Slides were scored semi-quantitatively based on staining intensity and distribution, and results were compared with clinical pathologic parameters. The mean follow-up was 56 months (range 1-169). Results: Seventy-three (48\%) of 151 breast tumors had loss of PTEN protein expression. On univariate analysis, loss of PTEN expression $(P=$ .034), stage $(P<.0001)$, node positive $(P<.0001)$, and tumor grade $(P=.002)$ were associated with disease-related death. Loss of PTEN expression also predicted lymph node metastasis $(P<.0001)$, and correlated with loss of estrogen receptor staining $(P$ $=.040)$. Loss of PTEN did not correlate with stage, tumor grade, disease recurrence, or loss of progesterone receptor [although a trend was seen $(P=$ .092). On multivariate analysis, stage $(P<.0001)$, lymph node metastasis $(P<.0001)$, and tumor grade $(P=.002)$ correlated with survival. Conclusion: Loss of PTEN protein expression occurs commonly in breast cancer and correlates with disease related death, lymph node metastasis, and loss of estrogen receptor staining. Our results support the
\end{abstract}

Copyright (C) 2001 by The United States and Canadian Academy of Pathology, Inc.

VOL. 14, NO. 7, P. 672, 2001 Printed in the U.S.A.

Date of acceptance: February 27, 2001.

Presented in part at the 89th Annual Meeting of the United States and Canadian Academy of Pathology, March 2000, New Orleans, Louisiana.

Dr. Ross is a consultant to Ventana Medical Systems, Inc., Tucson, Arizona.

Address reprint requests to: Jeffrey S. Ross, M.D., Department of Pathology, Mail Code 81, Albany Medical College, 47 New Scotland Avenue, Albany, NY 12208; e-mail: rossj@mail.amc.edu; fax: 518-262-8092. proposed role of PTEN as a candidate tumor suppressor in breast cancer and suggest a need for further study of this marker.

KEY WORDS: Breast cancer, Prognosis, PTEN. Mod Pathol 2001;14(7):672-676

Breast cancer is the most common cancer in women, accounting for $30 \%$ of all female malignancies, and is responsible for $16 \%$ of cancer-related deaths in this group, second only to lung cancer (1). Over the past few decades, there has been no significant decrease in incidence of this disease, and it continues to be a major cause of morbidity and mortality in the female population. This has stimulated broad public interest and research initiatives to aid in early detection and develop new therapies, specifically, agents directed at discrete cellular targets involved in regulating tumor growth and metastasis.

The PTEN gene (MMAC1-mutated in multiple advanced cancers) is a novel candidate tumor suppressor located on chromosomal band 10q23.3 (2). PTEN/ MMAC1 encodes a dual-specificity phosphatase that has recently been reported to dephosphorylate focal adhesion kinase (FAK), which results in inhibition of cell migration, spreading, and focal adhesion formation $(3,4)$. PTEN plays an important role in the modulation of the 1-phosphatidylinositol 3-kinase pathway (PI3K), which is involved in cell proliferation and survival (5). Specifically, in breast cancer, cell line analyses have shown that PTEN appears to suppress breast cancer growth by down-regulation of PI3K, with resultant G1 arrest and cell death $(6,7)$. Studies of embryonic stem cells have shown that cells featuring mutations of the PTEN gene exhibited an increased growth rate and displayed an advanced entry into S-phase (8). The accelerated $G_{1} / S$ transition was accompanied by down-regulation of $\mathrm{p} 27$, a major inhibitor of $G_{1}$ cyclin-dependent kinases. Overall, the results demonstrated that PTEN regulates cell cycle progression and cell survival.

Genetic alterations at the PTEN locus had been described in a variety of neoplasms, including primary central nervous system, breast, prostate, and 
bladder tumors, and non-Hodgkin's lymphoma (912). Additionally, germline mutations of the PTEN gene are associated with a rare, autosomal-dominant, familial cancer syndrome known as Cowden disease, which is associated with a 25 to $50 \%$ lifetime risk of developing breast cancer. Some studies have suggested that PTEN mutations may specifically lead to breast tumorigenesis in Cowden disease; however, other investigators could not confirm this association (13-15).

A few studies have investigated the role of PTEN in the tumorigenesis of sporadic breast cancer. These limited studies have found that PTEN gene mutations may be involved in a small subset of cases (16). Recent investigation by Ghosh et al. suggested that PTEN acts as a transcriptional repressor, inhibits the cell-mediated survival signaling pathway, and negatively regulates human breast carcinoma cell growth (17). The prognostic significance of PTEN protein loss in breast cancer, however, is not well established.

\section{MATERIALS AND METHODS}

Tissue blocks from the pathology archives at the Albany Medical Center were randomly selected from 151 women who underwent mastectomy or local excision for infiltrating mammary carcinoma between 1977 and 1995. The clinical records and follow-up information were also obtained where available. Hematoxylin- and eosin-stained slides were reviewed in each case to confirm the original diagnosis. Tumor stage (TNM system) and estrogen and progesterone steroid receptor hormone status (dextran charcoal competitive-binding assays and immunohistochemical measurements) were determined by review of the medical records. Tumors were graded according to the modified BloomRichardson grading system and separated into high grade (scores of 8 or 9) and non-high grade (score $<8$ ) categories (18). Follow-up data was available on 137 patients.

\section{Immunohistochemistry}

PTEN (monoclonal mouse anti-human PTEN [A2B1], Santa Cruz Biotech, Santa Cruz, CA) immunohistochemical staining was performed on the Ventana ES automated immunohistochemistry instrument (Ventana Medical Systems, Inc., Tucson, AZ) with an indirect biotin avidin system on $5 \mu \mathrm{m}$ formalin-fixed, paraffin-embedded sections from a representative block in each case. Tissue blocks were stored in ambient conditions in the pathology departments of both institutions 5 to 23 years before IHC procedures. All blocks were cut immediately before IHC. The slides were subsequently incubated with a biotinylated universal secondary antibody and an avidin horseradish peroxidase label (Ventana). After the development of color with diaminobenzidine substrate, the slides were counterstained with hematoxylin. Positive-control slides using prostatic adenocarcinoma with adjacent benign tissue were included with every batch. To confirm the specificity of the primary antibody, negative control slides were run using an isotypematched mouse IgG, (Sigma, St. Louis, MO) at the same concentration as that of the primary antibody. As an additional negative control, we evaluated PTEN immunostaining using the PC-3 prostate cancer cell line, and no staining was observed. The immunochemicals, incubation times, and final antibody concentration and dilutions are summarized as follows: monoclonal mouse antihuman PTEN, 32 minutes at $37^{\circ} \mathrm{C}$, dilution 1:10.

\section{Staining Interpretation}

Evaluation of PTEN expression was semiquantitative, based on staining intensity and distribution. Intensity was scored as strong, moderate, or weak. Distribution was scored as diffuse $(<50 \%$ tumor staining), regional (15 to 50\% tumor staining), and focal ( $<15 \%$ tumor staining). Tumors with intense to diffuse, intense to regional, intense to focal, and moderate to diffuse staining were considered positive for PTEN expression, whereas tumors with moderate to regional, moderate to focal, or weak staining with any distribution were considered negative.

\section{Statistical Analysis}

Statistical comparisons were carried out with STATA software (Computing Resource Center, Santa Monica, CA) using the $\chi^{2}$ test to determine the significance of the association between different variables. The comparison of PTEN expression, tumor stage, lymph node metastasis, tumor grade, disease recurrence, estrogen receptor staining (ER) status, and progesterone receptor (PR) status with each other and with survival were performed by univariate and multivariate analysis using the Cox proportional hazards model and the Kaplan-Meier test. The level of significance was set at .05.

\section{RESULTS}

There were 151 patients who ranged in age from 26-98 years (mean, 62). Of the 137 patients with follow-up information, the mean patient follow-up was 59 months (range, 1-169). There were 59 (43\%) patients who died and 78 (57\%) patients who were alive at the time of the completion of the study. Thirty-two (25\%) patients were stage I, 65 (50\%) were stage II, 11 (9\%) were stage III, and $21(16 \%)$ 
were stage IV. Of the 137 patients with lymph node status data, lymph node metastasis was present in $74(54 \%)$ of cases, whereas $63(46 \%)$ were node negative. One hundred four $(69 \%)$ of the total 151 infiltrating mammary carcinomas were ductal, and 47 (31\%) were lobular. There were 25 (25\%) highgrade and $75(75 \%)$ non-high grade tumors. Four ductal carcinomas were not graded because of small tumor size. Disease recurrence occurred in 28 of $127(22 \%)$ patients with available data. The ER and PR status was known in 89 and 78 patients, respectively. There were 64 (72\%) ER-positive tumors and 48 (62\%) PR-positive tumors.

\section{PTEN Expression by Immunohistochemistry}

Loss of PTEN protein expression was seen in 93 (48\%) of 151 breast tumors evaluated (Fig. 1). Loss of PTEN expression significantly correlated with disease-related death on univariate analysis (Fig. 2)
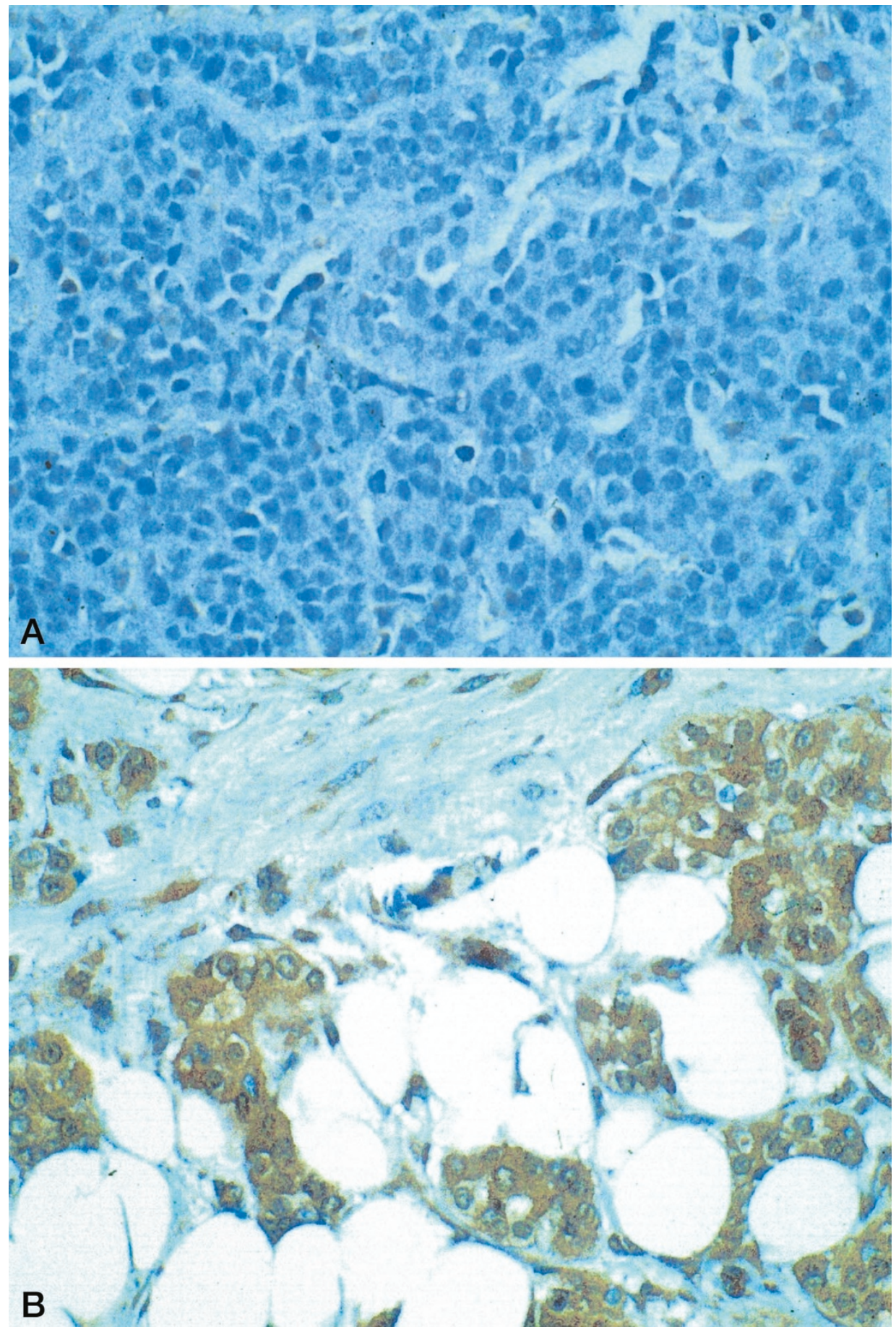

FIGURE 1. A, Breast tumor with no PTEN immunostaining $(\times 400)$. B, Breast tumor with positive PTEN staining $(\times 400)$. 
and also predicted lymph node metastasis, with 49 (60\%) of lymph node-positive cases showing PTEN protein loss $(P<.0001)$. Seventeen of $25(68 \%)$ tumors with negative ER status also showed loss of PTEN expression $(P=.040)$. There was no significant association between loss of PR status and PTEN expression, although a trend was observed $(P$ $=.092$ ). There was no correlation between the loss of PTEN expression and stage $(P=.391)$, disease recurrence $(P=.542)$, or tumor grade $(P=.817)$. See Table 1 .

\section{Survival Analysis}

On univariate analysis, loss of PTEN expression $(P=.034)$, stage $(P<.0001)$, node-positive status $(P$ $<.0001)$, and tumor grade $(P=.002)$ were all associated with disease-related death. On multivariate analysis, only stage $(P<.0001)$, node-positive status $(P=.008)$, and tumor grade $(P=.002)$ predicted survival. See Figure 2.

\section{CONCLUSIONS}

PTEN/MMAC-1 is a candidate tumor suppressor that appears to have a multifunctional role involved in cell proliferation, migration, and invasion (4). Alterations at the PTEN locus have been described in numerous malignancies, and recent reports have shown a regulatory role of PTEN in growth of breast carcinoma cells (17). Despite these findings, some studies failed to find loss of heterozygosity of PTEN in breast cancers and suggested that PTEN does not play a major role in breast tumorigenesis (19).

We have found significant loss of PTEN protein expression (48\%) in our breast cancer study group. This finding is similar to results of previous reports, including studies finding allelic loss of PTEN in 27 of $70(39 \%)$ and 17 of $42(40 \%)$ breast tumors, and another immunohistochemical study reporting weak or negative tumor staining in $33 \%$ of breast cancers analyzed (20-22). Overall, these findings

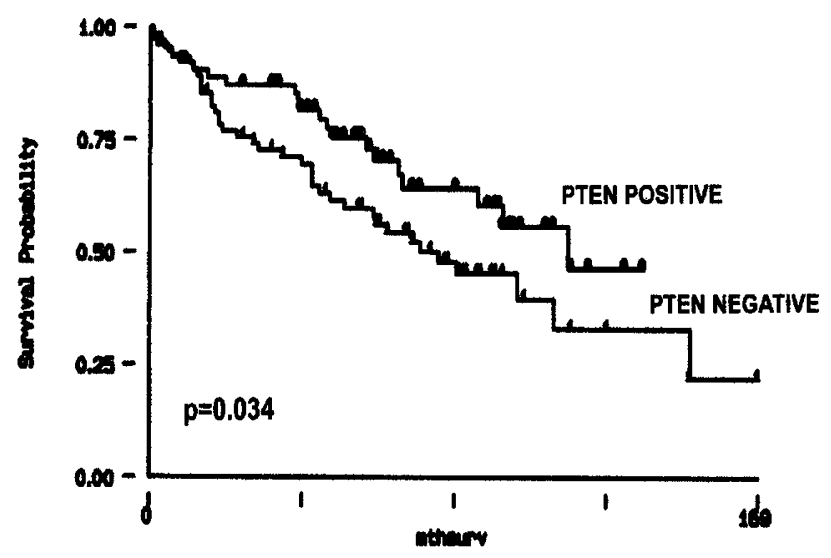

FIGURE 2. PTEN protein loss versus survival.
TABLE 1. PTEN Protein Loss and Outcome Variables

\begin{tabular}{lc}
\hline \multicolumn{1}{c}{ Variable } & Number of Cases $(P$ Value $)$ \\
\hline Disease-related death & $38 / 59(P=.034)$ \\
Node-positive status & $49 / 74(P<.0001)$ \\
High tumor grade & $13 / 25(P=.817), \mathrm{NS}$ \\
High stage $(3 \& 4)$ & $19 / 32(P=.391), \mathrm{NS}$ \\
Disease recurrence & $16 / 28(P=.542), \mathrm{NS}$ \\
ER negative & $17 / 25(P=.040)$ \\
PR negative & $19 / 30(P=.092), \mathrm{NS}$ \\
Tumor size $>5 \mathrm{~cm}$ & $10 / 18(P=.809), \mathrm{NS}$ \\
\hline
\end{tabular}

ER, estrogen receptor staining; NS, not significant.

indicate that PTEN alterations and protein loss are common in breast cancer.

Correlation of PTEN loss with outcome in breast neoplasia has not been well characterized in the literature, with few reports analyzing prognostic parameters. Perren et al. looked at outcome variables including tumor grade, tumor size, lymph node status, and ER/PR status. A study by Bose et al. evaluated loss of heterozygosity at 10q23 with stage, nuclear grade, DNA ploidy, and hormone receptor status. Comparisons with survival and disease recurrence were not performed in either study. Both analyses found association of PTEN loss with ER loss. Perren et al. additionally found significant association with PR loss. This finding is confirmed in our study with significant loss of PTEN expression in 17 of 25 ER-negative tumors and near-significant PTEN loss in PR-negative tumors. The underlying reason for this correlation is not clear, but PTEN expression loss appears to be linked to an aggressive ER/PR negative phenotype. We additionally report strong correlation of PTEN loss with lymph node metastasis, a finding not previously reported. Finally, of greatest significance in the present study was the association of PTEN protein loss with disease-related death, the first link of a major disease outcome variable in breast cancer with loss of expression of this tumor suppressor.

In conclusion, we have identified significant correlation of PTEN protein loss with adverse prognostic factors including steroid receptor hormonal loss, lymph node metastasis, and survival in breast cancer. These results concur with previous studies implicating PTEN as a candidate tumor suppressor in breast cancer. Further study of this gene in breast cancer patients appears warranted.

\section{REFERENCES}

1. Landis SH, Murray T, Bolden S, Wingo PA. Cancer statistics, 1998. CA Cancer J Clin 1998;48:6-29.

2. Steck PA, Pershouse MA, Jasser SA, Yung WKA, Lin H, Ligon $\mathrm{AH}$, et al. Identification of a candidate tumor suppressor gene, MMAC1, at chromosome 10q23.3 that is mutated in multiple advanced cancers. Nat Genet 1997;15:356-62.

3. Tamura M, Gu J, Matsumoto K, Aota S, Parsons R, Yamada KM. Inhibition of cell migration, spreading and focal adhesions by tumor suppressor PTEN. Science 1998;280:1614-7. 
4. Tamura M, Gu J, Takino T, Yamada KM. Tumor suppressor PTEN inhibition of cell invasion, migration, and growth: differential involvement of local adhesion kinase and p130cas. Cancer Res 1999;59:442-9.

5. Besson A, Robbins SW, Yong VW. PTEN/MMAC1/TEP1 in signal transduction and tumorigenesis. Eur J Biochem 1999; 263:605-11.

6. Weng LP, Smith WM, Dahia PLM, Ziebold U, Gil E, Lees JA, et al. PTEN suppresses breast cancer cell growth by phosphatase activity-dependent $\mathrm{G}_{1}$ arrest followed by cell death. Cancer Res 1999;59:5808-14.

7. Li J, Simpson L, Takahashi M, Miliaresis C, Myers MP, Tonks $\mathrm{N}$, et al. The PTEN/MMAC1 tumor suppressor induces cell death that is rescued by the AKT/protein kinase B oncogene. Cancer Res 1998;58:5667-72.

8. Sun H, Lesche R, Li DM, Ciliental J, Zhang H, Gao J, et al. PTEN modulates cell cycle progression and cell survival by regulating phosphatidylinositol 3,4,5-triphosphate and Akt/ protein kinase B signaling pathway. Proc Natl Acad Sci U S A 1999;96:6199-204.

9. Li J, Yen C, Liaw D, Podsypanina K, Bose S, Wang S, et al. PTEN, a putative protein tyrosine phosphatase gene mutated in human brain, breast, and prostate cancer. Science 1997;275:1943-7.

10. McMenamin ME, Soung P, Perera S, Kaplan I, Loda M, Sellers WR. Loss of PTEN expression in paraffin-embedded primary prostate cancer correlates with high Gleason score and advanced stage. Cancer Res 1999;59:4291-6.

11. Nakahara $Y$, Nagai $H$, Kinoshita $T$, Uchida T, Hatano S, Murate T, et al. Mutational analysis of the PTEN/MMAC1 gene in non-Hodgkin's lymphoma. Leukemia 1998;12:127780 .

12. Cairns P, Evron E, Okami K, Halachmi N, Esteller M, Herman JG, et al. Point mutation and homozygous deletion of PTEN/ MMAC1 in primary bladder cancers. Oncogene 1998;16: 3215-8.

13. Nelen MR, Kremer H, Konings IB, Schoute F, Van Essen AJ, Koch $\mathrm{K}$, et al. Novel PTEN mutations in Cowden disease: absence of clear genotype-phenotype correlations. Eur J Hum Genet 1999;7:267-73.

14. Carroll BT, Couch FJ, Rebbeck TR, Weber BL. Polymorphisms in PTEN in breast cancer families. J Med Genet 1999;36:94-6.

15. Lynch ED, Ostermeyer EA, Lee MK, Arena JF, Ji H, Dann J, et al. Inherited mutations in PTEN that are associated with breast cancer, cowden disease, and juvenile polyposis. Am J Hum Genet 1997;61:1254-60.

16. Rhei E, Kang L, Bogomolniy F, Federici MG, Borgen PI, Boyd J. Mutation analysis of the putative tumor suppressor gene PTEN/MMAC1 in primary breast carcinomas. Cancer Res 1997;57:3657-9.

17. Ghosh AK, Grigorieva I, Steele R, Hoover RG, Ray RB. PTEN transcriptionally modulates c-myc gene expression in human breast carcinoma cells and is involved in cell growth regulation. Gene 1999;235(1-2):85-91.

18. Elston CW, Ellis IO. Pathologic prognostic factors in breast cancer. I. The value of histologic grade in breast cancer: experience from a large study with long term follow-up. Histopathology 1991;19:403-10.

19. Chen ST, Yu SY, Tsai M, Yeh KT, Wang JC, Kao MC, et al. Mutation analysis of the putative tumor suppressor gene PTEN/MMAC1 in sporadic breast cancer. Breast Cancer Res Treat 1999;55:85-9.

20. Feilotter HE, Coulon V, McVeigh JL, Boag AH, DorionBonnet F, Duboue B, et al. Analysis of the 10q23 chromosomal region and the PTEN gene in human sporadic breast carcinoma. Br J Cancer 1999;79:718-23.

21. Bose S, Wang SI, Terry MB, Hibshoosh H, Parsons R. Allelic loss of chromosome 10q23 is associated with tumor progression in breast carcinomas. Oncogene 1998;17:123-7.

22. Perren A, Weng L, Boag A, Ziebold U, Thakore K, Dahia P, et al. Immunohistochemical evidence of loss of PTEN expression in primary ductal adenocarcinomas of the breast. Am J Pathol 1999;155:1253-60. 